\title{
7 Hitzebelastungen im Arbeitssetting: die Sicht der Arbeitsmedizin
}

\author{
Julia Schoierer, Hanna Mertes, Katharina Deering, \\ Stephan Böse-0'Reilly und Caroline Quartucci
}

C. Günster ||. Klauber | B.P. Robra | C. Schmuker | A. Schneider (Hrsg.) Versorgungs-Report Klima und Gesundheit. DOI 10.32745/9783954666270-7, @ MWV Medizinisch Wissenschaftliche Verlagsgesellschaft Berlin 2021

Mit fortschreitendem Klimawandel werden auch in Deutschland mehr Hitzeereignisse auftreten. Diese zunehmende Hitzebelastung kann (schwerwiegende) gesundheitliche Beschwerden verursachen, insbesondere bei vulnerablen Personengruppen. Zu diesen gehören auch Beschäftigte, die sogenannte Hitzetätigkeiten ausführen und/oder aufgrund ihrer Tätigkeit im Freien der Hitze direkt ausgesetzt sind. Dabei wird deren gesundheitliches Risiko durch weitere Cofaktoren wie z.B. die Art der Tätigkeit, der Berufskleidung und auch durch individuelle Faktoren wie Gesundheitszustand und Physiologie bestimmt.

Hitzebedingte Leistungsverluste und Arbeitsunfähigkeiten haben dementsprechend auch betriebs- und volkswirtschaftliche Konsequenzen. Im Sinne des Arbeits- und vorbeugenden Gesundheitsschutzes ist die Gesundheit der Beschäftigten vor Hitze zu schützen; hierfür sind je nach Tätigkeit und Arbeitsstätte verschiedene Maßnahmen umzusetzen.

As climate change progresses, more heat events will occur in Germany as well. The increasing heat stress can cause (serious) health problems, especially for vulnerable groups of people such as employees who perform so-called "heat activities" and/or are directly exposed to heat due to their outdoor work. Their health risk is determined by other cofactors such as the type of activity, work clothing and individual factors such as health status and physiology.

Heat-related loss of performance and incapacity to work have corresponding consequences for business and the economy. In the sense of occupational and preventive health protection, the employees' health must be protected from heat stress. Therefore various measures must be implemented depending on the activity and workplace.

\subsection{Gesundheitsrisiko durch Hitze}

In Deutschland wird es heißer, wie der Anstieg der mittleren Lufttemperatur zwischen 1881 und 2018 um $1,5^{\circ} \mathrm{C}$ zeigt. Das ist ein stärkerer Anstieg als der bisherige, weltweite Anstieg um 1, $0^{\circ} \mathrm{C}$ (Watts et al. 2018; Bissolli et al. 2019; MassonDelmotte et al. 2019). Das bedeutet, dass in Deutschland Hitzeereignisse, wie Hitzetage und -perioden sowie Tropennächte, zunehmen werden (Matthies-Wiesler 2019). Ein messbarer Anstieg dieser Ereignisse ist bereits jetzt zu beobachten (Masson-Delmotte et al. 2019; UBA 2019b). 
II Gesundheitliche Auswirkungen des Klimawandels und Herausforderungen für die medizinische Versorgung in Deutschland

Tab. 1 Darstellung der physiologischen Prozesse, die durch hohe Temperaturen in Gang gesetzt werden (Mora et al. 2017)

\begin{tabular}{llllll}
\hline Organe & Ischämie & $\begin{array}{l}\text { Hitze } \\
\text { Zytotoxizität }\end{array}$ & $\begin{array}{l}\text { Entzündungs- } \\
\text { reaktion }\end{array}$ & $\begin{array}{l}\text { disseminierte } \\
\text { intravasale } \\
\text { Koagulopathie* }\end{array}$ & $\begin{array}{l}\text { Rhabdo- } \\
\text { myolyse** }\end{array}$ \\
\hline Gehirn & $\mathrm{x}$ & $\mathrm{x}$ & $\mathrm{x}$ & $\mathrm{x}$ & \\
\hline Herz & $\mathrm{x}$ & $\mathrm{x}$ & $\mathrm{x}$ & $\mathrm{x}$ & \\
\hline Verdauungssystem & $\mathrm{x}$ & $\mathrm{x}$ & $\mathrm{x}$ & $\mathrm{x}$ & $\mathrm{x}$ \\
\hline Niere & $\mathrm{x}$ & $\mathrm{x}$ & $\mathrm{x}$ & $\mathrm{x}$ & $\mathrm{x}$ \\
\hline Leber & $\mathrm{x}$ & $\mathrm{x}$ & $\mathrm{x}$ & $\mathrm{x}$ & $\mathrm{x}$ \\
\hline Lunge & & $\mathrm{x}$ & $\mathrm{x}$ & $\mathrm{x}$ & \\
\hline Pankreas & $\mathrm{x}$ & & $\mathrm{x}$ & & \\
\hline
\end{tabular}

Ist kein $\mathrm{x}$ in den Feldern enthalten, sind keine entsprechenden Studien in das Review von Mora et al. (2017) eingeflossen.

*sekundäre Erkrankung, die von einer systemischen, exzessiven Aktivierung der Blutgerinnung ausgelöst wird

**Auflösung quergestreifter Muskelfasern

Extreme Hitzeereignisse stellen für den menschlichen Körper eine hohe Belastung dar (Kenny et al. 2010, 2018). Je nach persönlicher Disposition ist es möglich, dass sich bestehende Erkrankungen verschlechtern oder neue, schwerwiegende Gesundheitsprobleme entstehen (Kenny et al. 2020). Diese können auch zum Tode führen, wie Auswertungen der Morbiditäts- und Mortalitätsdaten aus Deutschland zeigen, die die Zunahme von Krankenhauseinweisungen und Sterbefällen während Hitzeereignissen belegen (Gabriel u. Endlicher 2011; Steul et al. 2018; an der Heiden et al. 2019, 2020). Dabei zeigen die Studien, dass besonders ältere bzw. hochaltrige Menschen durch Hitze gesundheitlich betroffen sind (Matthies et al. 2008; Kenny et al. 2010; Herrmann et al. 2019; Meade et al. 2020). Aber auch quer durch die Bevölkerung wird Hitze zunehmend als gesundheitliche Belastung wahrgenommen, wie eine Studie des Bundesministeriums für Umwelt, Naturschutz und nukleare Sicherheit (BMU) und des Umweltbundesamtes (UBA) zeigt. Demnach fühlten sich 2012 ein knappes Drittel der Befragten von Hitzeperioden in ihrem körperlichen Wohlbefinden beeinträch- tigt - 2016 waren es 50\% (BMU 2013; BMU u. UBA 2017)

Weltweit sind die Forschungen zu gesundheitlichen Hitzefolgen in den letzten Jahren intensiver geworden. Damit wurden Erkenntnisse gewonnen, wie Hitze auf den menschlichen Körper wirken kann und welche Personengruppen besonders von Hitze betroffen sind. So haben zum Beispiel Mora und Kollegen in ihrer Studie „Twenty-seven ways a heat wave can kill you: Deadly heat in the era of climate change“ die Auswirkungen von Hitze auf den menschlichen Körper dargestellt (s. Tab. 1) (Mora et al. 2017). Sie zeigen in ihrem Review, dass hohe Temperaturen eine Reihe physiologischer Prozesse in Gang setzen können, die zu hitzebedingten gesundheitlichen Problemen oder sogar zum Tod führen können. Pathologisch betroffen sind dabei nicht nur das Herz-Kreislaufund das Atmungssystem, sondern u.a. auch das Verdauungssystem, die Leber und die Niere. Bei Hitze besteht also ein veritables Risiko, gesundheitliche Probleme zu entwickeln, siehe hierzu auch Kenny et al. (2020).

Das Risiko für hitzebedingte Gesundheitsprobleme wird neben dem Alter durch weitere 


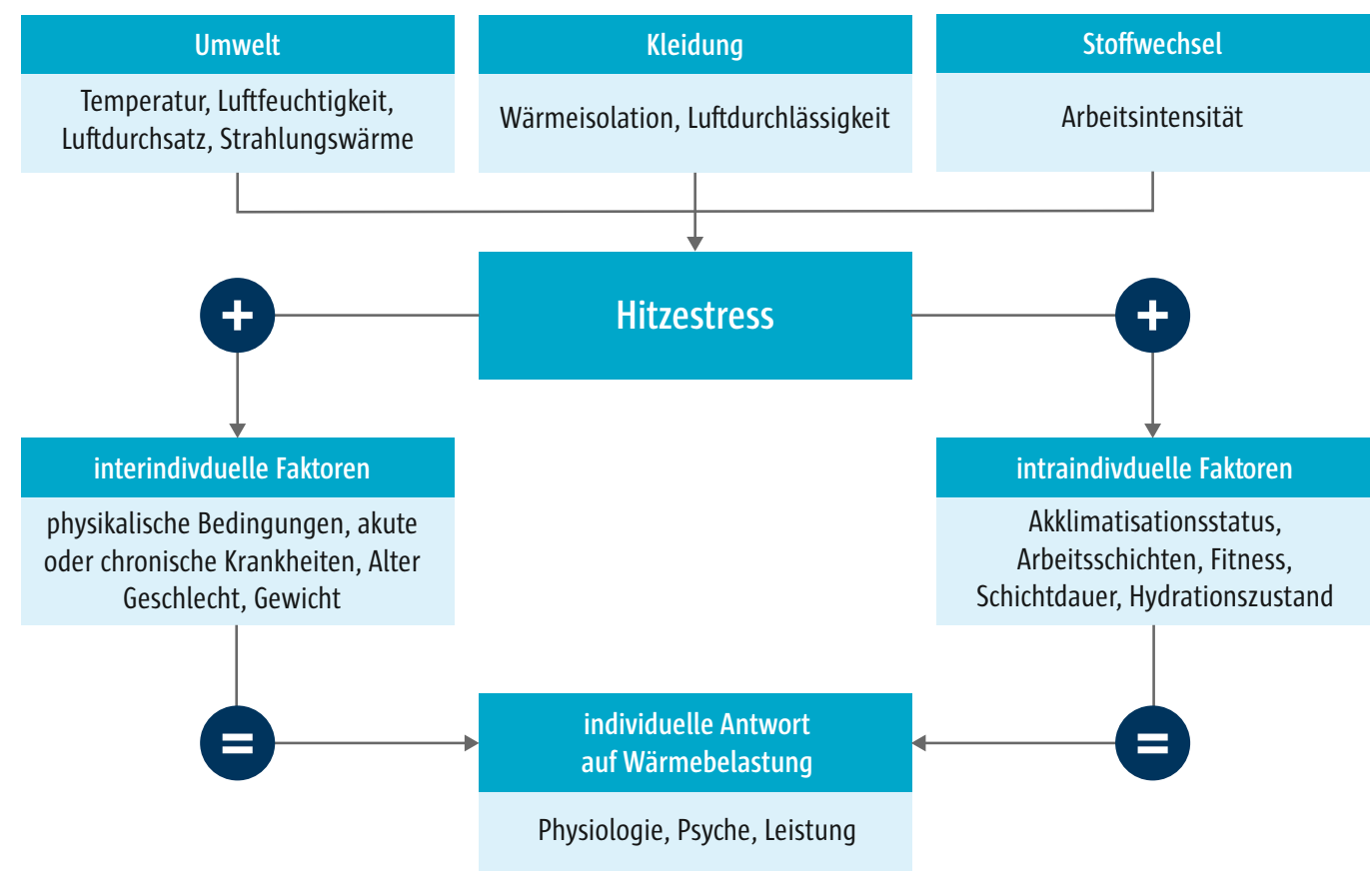

Abb. 1 Faktoren für Hitzestress (modifiziert nach Kenny et al. 2020)

Faktoren erhöht, wie die individuelle Physiologie, chronische Erkrankungen, die Einnahme von Medikamenten sowie die Art der Exposition gegenüber Hitze, z.B. während der Arbeit (Kenny et al. 2010, 2020). Dabei können Berufstätige auf zwei Wegen von Hitze betroffen sein: Zum einen durch klimabedingte Hitzeereignisse, zum anderen durch ihre Tätigkeit, die durch die Gefährdungsbeurteilung als Hitzearbeit definiert wird und/oder deren Hitzeexposition möglicherweise durch den Klimawandel noch verstärkt wird (Kjellstrom et al. 2016; Levi et al. 2018; Binazzi et al. 2019; Kjellstrom et al. 2019; Spector et al. 2019).

Wie hoch die Belastung durch Hitze während der Arbeit ist, hängt also zusätzlich von der Tätigkeit (Tragen von Berufskleidung, Schwere der Arbeit, Dauer und Art der Exposition) sowie von der individuellen Physiologie und Anpassungsfähigkeit des Beschäftigten ab (z.B. chronische Erkrankungen, Übergewicht, Alter, körperliche Fitness) (Jacklitsch et al. 2016; Kenny et al. 2020).
Neben den individuellen gesundheitlichen Belastungen können hitzebedingte Gesundheitsprobleme aufgrund der Kosten für die Gesundheitsversorgung oder der Reduzierung von Leistungsfähigkeit auch zu erheblichen wirtschaftlichen Folgen führen (Lundgren et al. 2013; Kjellstrom et al. 2016; Flouris et al. 2018; Gao et al. 2018; Spector et al. 2019). So stellen Kenny et al. (2020) in ihrem Buchbeitrag „Climate Change and Heat Exposure: Impact on Health in Occupational and General Populations" eine umfassende Sichtweise auf den komplexen $\mathrm{Zu}$ sammenhang von Hitze und Gesundheit für Beschäftigte, aber auch für die Allgemeinbevölkerung dar (s. Abb. 1). Anhand von Abbildung 1 lassen sich sehr gut die unterschiedlichen Quellen von Hitzestress - Umweltbedingungen/Umgebung, Kleidung, durch Stoffwechselprozesse erzeugte Wärme - sowie deren beeinflussende Faktoren (inter- und intraindividuell) erkennen, deren Zusammenwirken letztlich die Reaktion auf die Hitzebelastung bedingt hinsichtlich 
II Gesundheitliche Auswirkungen des Klimawandels und Herausforderungen für die medizinische Versorgung in Deutschland

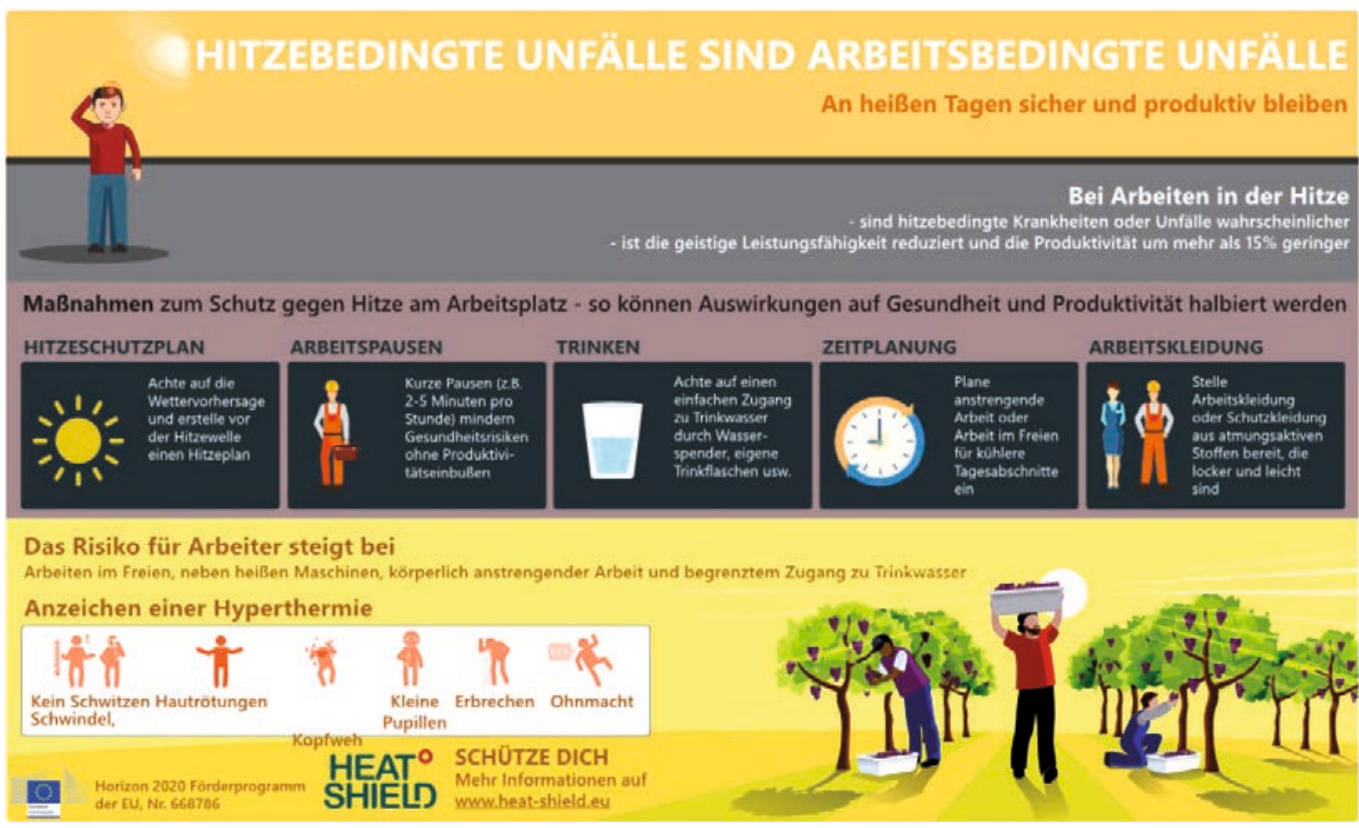

Abb. 2 Beispielhafte Informationsgrafik des europäischen Projekts Heat-Shield zur Vermeidung hitzebedingter Erkrankungen bei Beschäftigten in Europa (https://heatshield.zonalab.it/infographics.php?lang=de)

physiologischer, psychophysischer und leistungsbezogener Effekte. Interindividuelle Faktoren sind hierbei persönliche körperliche Faktoren, die zwischen verschiedenen Individuen unterschiedlich ausgeprägt sind und sich in ihrer Ausprägung nicht kurzfristig ändern lassen. Intraindividuelle Faktoren sind Faktoren, die einem persönlichen Verhalten zugrunde liegen und verändert werden können.

Auch im Rahmen des europäischen Projekts HEAT-SHIELD (www. heat-shield.eu) mit zahlreichen wissenschaftlichen Institutionen sowie politischen und kommunalen Einrichtungen wird der Einfluss der in Europa zunehmenden thermischen Belastung auf unterschiedliche Arbeitsbereiche untersucht. Dabei stehen die Bereiche Handwerk, Bau, Tourismus, Landwirtschaft und Transport bezüglich gesundheitlicher und ökonomischer Effekte im Fokus (Morabito et al. 2019). Die Ergebnisse werden sowohl in wissenschaftlichen Publikationen als auch in allgemeinverständlichen Handlungsempfehlungen dargestellt und richten sich an die genannten Zielgruppen zur Prävention hitzebedingter Gesundheitsprobleme (s. Abb. 2) (Morris et al. 2020).

Auch in Deutschland liegen Instrumente für Arbeitgeber und Beschäftigte zur Anpassung an Hitzeereignisse vor, z.B. „Empfehlungen für heiße Sommertage in Arbeitsstätten" (2006) sowie eine „Arbeitsstättenregel ASR A3.5 Raumtemperatur" von der Bundesanstalt für Arbeitsschutz und Arbeitsmedizin (2018). Beide Instrumente zum Hitzeschutz von Beschäftigen sind im Klimavorsorgeportal aufgelistet und können frei heruntergeladen werden (www.klivoportal.de).

Im Arbeitssetting werden Hitzebelastungen nach dem Arbeitsschutzgesetz und den darin geforderten Gefährdungsbeurteilungen bewertet (s. Kap. 7.2.1 und 7.2.2). Gleichfalls können weitere Faktoren das Risiko für die Entwicklung hitzebedingter Gesundheitsprobleme erhöhen. Diese werden in den Kapiteln 7.2.3 und 7.2.4 anhand der Beispiele individueller und sozioökonomischer Faktoren näher erläutert. 


\subsection{Risikofaktoren für eine erhöhte Hitzebelastung am Arbeitsplatz}

Um eine erhöhte Hitzeexposition am Arbeitsplatz zu vermeiden, ist der Arbeitgeber nach dem Arbeitsschutzgesetz verpflichtet, die Arbeitsplätze hinsichtlich der auftretenden Gefährdungen $\mathrm{zu}$ beurteilen. In diese Gefährdungsbeurteilung fließen auch Faktoren ein, die das Raumklima und somit auch hohe Temperaturen während der Sommermonate einschließen (Deutsche Gesetzliche Unfallversicherung e.V., DGUV 2010). Die Arbeitskleidung und die dadurch möglicherweise erhöhte/verstärkte Hitzebelastung werden dabei ebenso berücksichtigt.

\subsubsection{Hitze und Umgebungstemperatur im Innen- und Außenraum}

Aus arbeitsmedizinischer Perspektive wird der Schutz der Beschäftigten vor Hitze bisher vor allen Dingen in Zusammenhang mit Vorgängen beschrieben, bei denen es zur Abgabe von Wärme kommt. Hierzu gehören klassischerweise z.B. Schweißarbeiten. Aber auch Tätigkeiten in anderen Bereichen können zu gesundheitlichen Gefährdungen durch Hitze führen und sollten in der Gefährdungsbeurteilung entsprechend bedacht werden. Beispiele hierfür sind (Deutsche Gesellschaft für Arbeitsmedizin und Umweltmedizin e.V. DGAUM 2012):

- Eisen- und Stahlherstellung

- Metallbe- und -verarbeitung

- Glas- und Keramikproduktion

- Spüleinrichtungen

- Küchen und Wäschereien

Liegt mindestens einer der in der untenstehenden Aufzählung genannten Parameter vor, ist eine arbeitsmedizinische Vorsorge notwendig. Beurteilt werden hierfür Lufttemperatur, Luftfeuchte, Flüssigkeitsaufnahme sowie Wärmestrahlung im Gesicht ( $\$ 4$ Absatz 1 in Verbindung mit Anhang Teil 3 Absatz 1 Nummer 1 Arb-
MedVV; siehe hierzu auch AMR [arbeitsmedizinische Regel] 13.1). Wenn diese Parameter allerdings durch die Anwendung von technischen (z.B. Jalousien, Klimaanlagen) oder organisatorischen (z.B. flexible Gestaltung der Arbeitszeit, Verkürzung der Tätigkeit in den Bereichen mit hoher Temperatur) Arbeitsschutzmaßnahmen ausreichend verändert werden können (s. Kap. 7.4.1), ist keine arbeitsmedizinische Vorsorge mehr notwendig.

\section{Parameter, um eine besondere Gefährdung durch Hitze während der Tätigkeit zu beurteilen \\ n Lufttemperatur über $45^{\circ} \mathrm{C}$ und Beschäftigungsdauer $>15$ Min. \\ - Lufttemperatur über $30^{\circ} \mathrm{C}$ mindestens vier Stunden pro Schicht und gleichzeitig hohe Luftfeuchte (ge- kennzeichnet beispielsweise durch feuchte oder nas- se Haut) \\ - Flüssigkeitsaufnahme über vier Liter pro Schicht \\ - Wärmestrahlung im Gesicht unerträglich}

Eine weitere Hilfestellung, um das Raumklima an Arbeitsplätzen (keine Anwendung auf Arbeitsplätze in Fahrzeugen und im Freien) beurteilen und Gesundheitsbelastungen vorbeugen zu können, gibt die DGUV-Information (DGUV 2016) „Beurteilung des Raumklimas: Gesund und fit im Kleinbetrieb“. Hiermit soll Unternehmen eine Hilfestellung gegeben werden, um raumklimatische Probleme zu erkennen, zu verringern und, wenn möglich, selbst zu beseitigen. Die Checkliste fragt dabei die Lufttemperatur und -feuchtigkeit, die Flüssigkeitsaufnahme, die Wärmestrahlung sowie das subjektive Befinden des Beschäftigten ab. Die Ergebnisse liegen entweder im grünen, gelben oder roten Bereich und können ggf. Maßnahmen nach sich ziehen. Sind die Beurteilung des Raumklimas oder entsprechende Maßnahmen für den Arbeitgeber schwer durch- oder umsetzbar, ist eine Raumklimaanalyse durch entsprechend ausgebildetes Personal (Sicherheitsfachkräfte, Betriebsärzte, Berufsgenossenschaften) durchzuführen. 
II Gesundheitliche Auswirkungen des Klimawandels und Herausforderungen

für die medizinische Versorgung in Deutschland

In Außenbereichen sind hauptsächlich Beschäftigte aus der Land- und Forstwirtschaft, dem Gartenbau oder bei der Durchführung von Bauarbeiten betroffen. Sie sind der Hitze direkt und ungeschützt ausgesetzt (DGAUM 2012).

\subsubsection{Hitzebelastung aufgrund von Arbeits- kleidung}

Um den Einfluss der Arbeitskleidung auf die thermische Belastung der Beschäftigten zu untersuchen, sind alle Faktoren zu berücksichtigen, die die metabolische Wärmeproduktion des menschlichen Körpers und seinen Wärmeaustausch mit der Umgebung beeinflussen. Diese sind in der Leitlinie „Arbeit unter klimatischer Belastung: Hitze“ definiert als:

- Arbeitsenergieumsatz (Arbeitsenergieumsatz = Gesamtenergieumsatz - Grundenergieumsatz), d.h. metabolische Wärmeentwicklung (zur Einordnung des Arbeitsenergieumsatzes während einer 8-stündigen Arbeitsschicht siehe DIN 33403 T3 oder DGAUM 2012.)

- Bekleidungsisolation

- Expositionsdauer

\section{Definition Schutzkleidung}

Arbeitsrechtlich sind die Untergruppen Berufskleidung, Dienstkleidung und Schutzkleidung zu unterscheiden. Schutzkleidung ist eine Arbeitskleidung, die aus Gründen des Arbeitsschutzes vom Arbeitnehmer zu tragen ist, z.B. im Gesundheitswesen, bei der Feuerwehr, in der Gärtnerei- und Forstwirtschaft oder in technischen Bereichen.

Es ist davon auszugehen, dass einige Formen der Schutzkleidung, z. B. zum Schutz vor Infektionen im Gesundheitswesen, vor allem bei erhöhten Raumtemperaturen zu verstärktem Schwitzen und zu rascherer körperlicher Ermüdung führen (Dorman u. Havenith 2009; Jacklitsch et al. 2016) (s. Definition Schutzklei- dung). Die reduzierte Luftströmung unter der Schutzkleidung verringert die Abführung der Körperwärme und der Körper kann überhitzen (DGAUM 2012). Neben der eigenen Gesundheit wird aber auch die Qualität der Arbeit beeinträchtigt. Dadurch, dass die Schutzkleidung auch in Umgebungen getragen werden muss, in denen es durch unzureichende oder fehlende Klimatisierung zu Temperaturen von $30^{\circ} \mathrm{C}$ oder mehr kommen kann, ist mit einer erheblichen thermischen Beanspruchung der Arbeitnehmer zu rechnen (Klenk et al. 2010). Bei (hitzebedingten) Problemen mit der Schutzkleidung ist dann der zuständige Betriebsarzt zu konsultieren. Dieser kann die individuelle Gefährdung aufgrund des Gesundheitszustandes des Beschäftigten und unter Berücksichtigung weiterer Faktoren wie Akklimatisation, Körpergewicht, Ernährungszustand, Konstitution, Trainingszustand und psychosozialen Faktoren beurteilen (DGAUM 2012).

\subsubsection{Hitzebelastung aufgrund individueller Risikofaktoren}

Alter

Da altersbedingt die Thermoregulationsfähigkeit des Körpers abnimmt, weil sich die Transpiration, der Blutfluss zur Haut und die kardiovaskuläre Leistungsfähigkeit vermindern, können sich ältere Menschen, abhängig von individueller Physiologie und Fitness, nicht mehr in dem Maße an Hitze anpassen wie jüngere Menschen (Best et al. 2012; Schoierer et al. 2019). Das Vorkommen weiterer Risikofaktoren, wie bspw. chronische Erkrankungen und (eine damit einhergehende) Medikamenteneinnahme, erhöhen das Risiko für hitzebedingte Gesundheitsprobleme, da sich beispielsweise die Symptomatik der bestehenden Vorerkrankung (deutlich bis lebensgefährlich) verschlechtern kann. Dies trifft bereits auf Menschen ab 6o Jahren zu, weshalb somit ein in den letzten Jahren gewachsener Anteil der beschäf- 
tigten Bevölkerung zur Risikogruppe gehört. Während im Jahr 2008 beispielsweise 8\% der Menschen zwischen 65 und 69 Jahren berufstätig waren, lag dieser Wert im Jahr 2018 bei 17\% (Destatis 2020).

Der Schutz älterer Beschäftigter vor Hitzeerkrankungen gewinnt folglich aus Sicht der Arbeitsmedizin zunehmend an Bedeutung, da die körperliche Fitness sowie der Gesundheitszustand eine wichtige Rolle in der körperlichen Resilienz gegenüber Hitze spielen (Lundgren et al. 2013).

\section{Chronische Erkrankungen}

Studien haben zum Beispiel ein erhöhtes Risiko für das Auftreten kardialer Thrombosen im Zusammenhang mit Dehydration bei hohen Temperaturen gezeigt (Näyhä 2005). Eine belgische Studie (Nawrot et al. 2005) identifiziert die endotheliale Dysfunktion als möglichen Mechanismus für die kardiovaskulären Wirkungen. Eine Hitzewelle in Kalifornien im Jahr 2006 führte zu einem starken Anstieg der Krankenhauseinweisungen aufgrund von Herz-Kreislauf- und anderen Erkrankungen (Knowlton et al. 2009; Takaro et al. 2013).

In weiteren Studien konnte zudem gezeigt werden, dass es durch die klimabedingte Temperaturerhöhung zu Nierenerkrankungen kommen kann (Johnson et al. 2019). Der Hitzschlag kann eine wichtige Rolle bei der Verursachung von akuten Nierenerkrankungen spielen, was zu einem erhöhten Risiko für akute Nierenverletzungen durch die Auflösung quergestreifter Muskelfasern (Rhabdomyolyse) oder hitzeinduzierte entzündliche Nierenschäden führt (s. Tab. 1). Wiederkehrende Hitze und dadurch bedingte Dehydration können zu chronischen Nierenerkrankungen (chronic kidney disease $=$ CKD) führen. Hitzestress und Dehydration spielen auch eine Rolle bei der Bildung von Nierensteinen. Unzureichende Hydratation kann das Risiko für wiederkehrende Harnwegsinfektionen erhöhen (Mora et al. 2017).
Bei medikamentös behandelten chronischen Erkrankungen können sich die Medikamente auf das Durstgefühl, das Trinkverhalten und den körpereigenen Wasserhaushalt auswirken. Ebenso kann Hitze die Wirkung und Nebenwirkungen von Medikamenten beeinflussen (Vassallo u. Delaney 1989; Cuddy 2004; Nordon et al. 2009; Westaway et al. 2015; Kalisch Ellett et al. 2016; Mangoni et al. 2016, 2017; Kiesel et al. 2018; Universitätsklinikum Heidelberg 2020).

\section{Übergewicht}

Menschen mit einem hohen Übergewicht (BMI $30+$ ) sind durch Hitze zusätzlich belastet. Das Fettgewebe fungiert als Isolationssperre, das den gesunden Wärmehaushalt des Körpers empfindlich stören kann. Um die nötige Körperkerntemperatur von circa $37^{\circ} \mathrm{C}$ konstant zu halten, muss das Herzkreislaufsystem verstärkt Blutvolumen in die Außenbereiche unseres Körpers pumpen. Dies belastet das Herzkreislaufsystem zusätzlich (Meade et al. 2020).

Des Weiteren gehen mit Übergewicht eine geringere Fitness und oftmals Folgeerkrankungen einher, wie Erkrankungen des Herzkreislaufsystems, Diabetes mellitus oder Erkrankungen des Bewegungsapparates. Diese Folgeerkrankungen erschweren die Anpassung an Hitze noch einmal zusätzlich (Meade et al. 2020).

\subsubsection{Hitzebelastung aufgrund sozio- ökonomischer Faktoren}

Das gesundheitliche Risiko am Arbeitsplatz kann weiterhin davon beeinflusst werden, inwiefern die Beschäftigten im privaten Bereich gegenüber Hitze exponiert sind und sich in ihren Erholungszeiten zu Hause regenerieren können. Zu berücksichtigende Faktoren sind unter anderem eine ländliche oder städtische 
II Gesundheitliche Auswirkungen des Klimawandels und Herausforderungen

für die medizinische Versorgung in Deutschland

Wohnumgebung, die Süd-/Westausrichtung der Wohnung, eine Lage der Wohnung im Dachgeschoss, eine unzureichende Dämmung bzw. auch fehlende, effektive Verschattungsmöglichkeiten (UBA 2019a). Hohe Innenraumtemperaturen können den nächtlichen Erholungsschlaf empfindlich stören und z.B. zu Erschöpfung, Konzentrationsschwäche und allgemeinem Unwohlsein am nächsten Tag führen (HARVARD Kennedy School 2018; Zheng et al. 2019). Dies kann ebenso die Leistungsfähigkeit während der Arbeit als auch das Unfallrisiko beeinflussen (Rameezdeen u. Elmualim 2017). Der Erholungswert in ländlichen Gebieten ist in Bezug auf Hitzeereignisse höher als für Bewohner in der Stadt, da sich Städte aufgrund des Wärmeinseleffektes stärker aufheizen und dadurch ein teils sehr deutlicher Temperaturunterschied zwischen städtischen und ländlichen Räumen entstehen kann (Brasseur et al. 2017). Damit gewinnen auch sozioökonomische Faktoren an Bedeutung, da die zur Verfügung stehenden finanziellen Mittel mit der Wohnsituation assoziiert sind und auch die Umsetzbarkeit entsprechender Hitzeanpassungsmaßnahmen (Umzug, Installation von Rollos etc.) bestimmen. So zeigt bspw. die Analyse der Stadt Berlin, dass die bioklimatische Belastungssituation in sozial schwächeren Stadtteilen höher ausfällt (Senatsverwaltung für Stadtentwicklung und Wohnen 2015).

\subsection{Hitze und die Folgen für die Arbeits- welt}

\subsubsection{Komplexität von Hitze, Gesundheit und Volkswirtschaft}

Ursachen von Hitze sowie die Konsequenzen verstärkende Faktoren sind sowohl für den Einzelnen als auch für die Gesellschaft äußerst komplex miteinander verbunden, wie die vorangegangenen Kapitel gezeigt haben. Kjellstrom et al. (2016) haben diese Komplexität anhand eines Flussdiagramms dargestellt (s. Abb. 3).
Dabei wird deutlich, dass Hitze sowohl negative Konsequenzen für die Gesundheit der Bevölkerung als auch für die Volkswirtschaft hat.

\subsubsection{Gesundheit und Produktivitätsverlust durch Hitze}

Diesen Ansatz verfolgt auch das europäische Projekt HEAT-SHIELD, welches den Einfluss von klimawandelbedingter Hitze auf die Arbeitswelt in Europa untersucht und den Produktivitätsverlust durch die Umgebungswärme darstellt. Dabei wird zum einen der beobachtete, zum anderen der projizierte (mittlere) Prozentsatz (Annahme: stärkstes Emissionsszenario [RCP8.5]) der verlorenen Sommerarbeitszeit aufgrund von Hitzeeinwirkung unter sonnigen Bedingungen betrachtet. Während im Zeitraum 1981 bis 2010 nur in wenigen Gegenden Europas mehr als 5\% der Arbeitsleistung aufgrund zu hoher Temperaturen verloren gingen und nur selten ein Verlust von $25 \%$ verzeichnet werden konnte, zeigt die Projektion, dass insbesondere in den Anrainerstaaten des Mittelmeeres dieser Wert im Zeitraum von 2070 bis 2099 auf 50\% steigen könnte. Auch zeigt die Projektion eine Zunahme des Arbeitsverlustes in Deutschland, insbesondere in den südlichen Regionen (Casanueva et al. 2020).

\subsubsection{Zunahme der Arbeitsunfähigkeitsfälle durch Hitze und Sonnenlicht (ICD-T67)}

Einige Studien belegen mittlerweile den $\mathrm{Zu}$ sammenhang von extremer Hitze und ernsthaften Erkrankungsfällen z.B. bei Außenarbeitern (Gubernot et al. 2015; Kjellstrom et al. 2018; Riley et al. 2018). In Deutschland sind diese Fälle unter dem ICD-10-Diagnoseschlüssel T67, Schäden durch Hitze und Sonnenlicht, klassifiziert. Hierzu gehören Hitzeschlag, Sonnenstich, Hitzekollaps, Hitzekrampf, Hitzeerschöpfung (durch Wasserverlust), Hitzeerschöpfung (durch Salzverlust), Hitzeerschöp- 


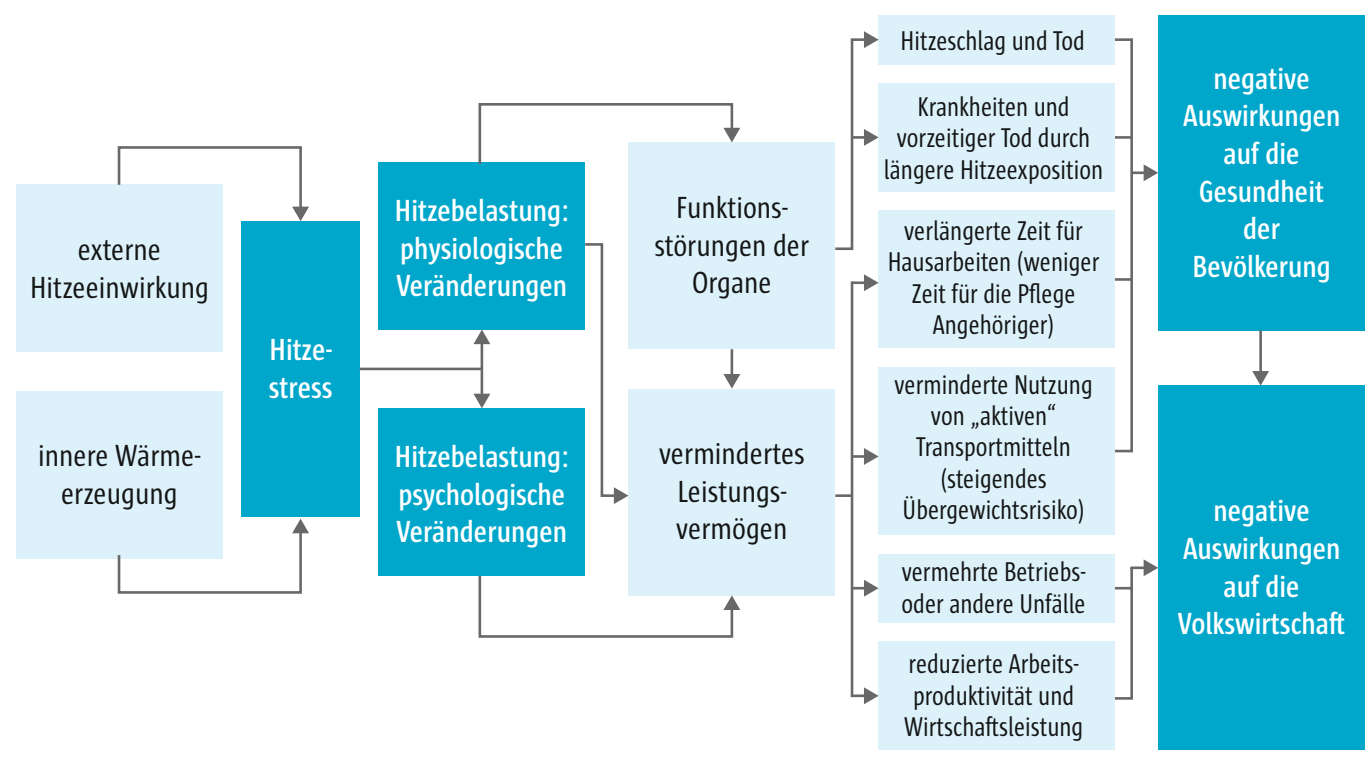

Abb. 3 Flussdiagramm (modifiziert nach Kjellstrom et al. 2016, mit freundlicher Genehmigung von Annual Reviews, Inc.)

fung (nicht näher bezeichnet), passagere Hitzeermüdung, Hitzeödem, sonstige Schäden durch Hitze und Sonnenlicht, Schäden durch Hitze und Sonnenlicht (nicht näher bezeichnet). Die Ergebnisse der Krankheitsartenstatistik der gesetzlichen Krankenversicherung aus dem Jahr 2018 (Bundesgesundheitsministerium, BMG 2018b) zeigen, dass unter dem ICD1o T67 insgesamt 79.177 Arbeitsunfähigkeitstage verzeichnet sind, welche von 19.921 Fällen durch Pflichtmitglieder in Anspruch genommen wurden.

Es ist ersichtlich, dass die Fehltage durch „Schäden durch Hitze und Sonnenlicht" kontinuierlich steigen (s. Abb. 4). Besonders in den Hitzejahren 2010, 2013, 2015 und 2018 sind viele Arbeitsunfähigkeitstage verzeichnet. In dieser Statistik nicht erfasst sind die Personen mit Krankheiten durch Hitze, die nicht bei einem Arzt vorstellig wurden oder die von dem behandelnden Arzt nicht als ICD-T67 Fälle eingestuft worden sind, beispielsweise Übelkeit oder Kopfschmerzen, welche auch hitzebedingt auftreten können.
Somit ist von einer höheren Dunkelziffer auszugehen. Dies bedeutet nicht nur eine ökonomische Belastung der Gesellschaft durch die Abrechnung der Krankheitstagegelder und Inanspruchnahme von ärztlicher Beratung, sondern auch ein durch die Arbeitsausfälle verursachter ökonomischer Schaden bei den jeweiligen Firmen (Vanos et al. 2019). Bisherige Studien, die den wirtschaftlichen Verlust aufgrund von Fehltagen der Beschäftigten durch Hitze quantifizieren, liegen bisher nur für wenige Berufsgruppen vor und hier in erster Linie für Außenarbeiter.

Die Ergebnisse lassen sich oftmals nicht oder nur bedingt vergleichen, zeigen jedoch eine erste Richtung der Forschungsentwicklung an.

In Anbetracht des Klimawandels und der $\mathrm{Zu}-$ nahme der Hitzetage kann von einer weiteren Steigerung der Arbeitsunfähigkeitstage bzw. der Krankheitsfälle aufgrund von Hitze ausgegangen werden, sofern nicht umfangreiche Präventionsmaßnahmen für die einzelnen Gruppen entwickelt bzw. angeboten werden. 
II Gesundheitliche Auswirkungen des Klimawandels und Herausforderungen für die medizinische Versorgung in Deutschland

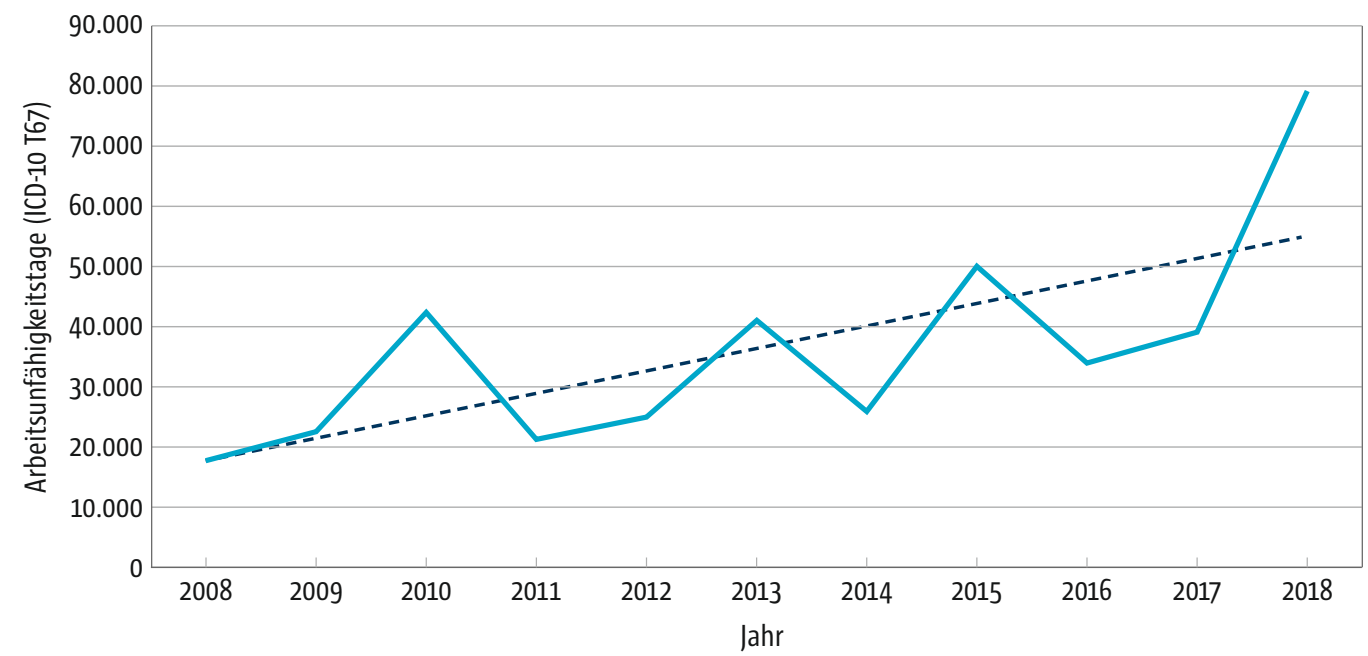

Abb. 4 Darstellung der Arbeitsunfähigkeitstage nach ICD-10 T67 der Jahre 2008 bis 2018 (Daten aus BMG 2018a)

\subsection{Arbeitsmedizinische Prävention}

Im Arbeitssicherheitsgesetz $($ ASiG $\mathbb{3} 3)$ ist festgelegt, dass der Betriebsarzt den Arbeitgeber bei der Erstellung der Gefährdungsbeurteilung nach dem Arbeitsschutzgesetz (ArbSchG $\mathbb{S} 5$ ) unterstützen soll. Eine arbeitsmedizinische Pflichtvorsorge wird notwendig, wenn innerhalb einer Gefährdungsbeurteilung eine mögliche Gefährdung durch extreme Hitze festgestellt wurde. Hierzu hat die DGAUM bereits eine umfangreiche und hilfreiche Zusammenstellung veröffentlicht, an der sich die nun folgenden Ausführungen orientieren (DGAUM 2012).

\subsubsection{Rechtliche Grundlagen}

Der Schutz der Beschäftigten vor hitzebedingten Gesundheitsproblemen ist in der Arbeitsstättenregel (ASR) 3.5 „Raumtemperatur“ festgelegt (BAuA 2018). Der Arbeitgeber muss darauf achten, dass die notwendigen baulichen Voraussetzungen an den sommerlichen Wärmeschutz gegeben sind. In vielen Bereichen kann so die Hitzebelastung durch technische Möglichkeiten verringert werden. Wenn die Außen- lufttemperatur $+26^{\circ} \mathrm{C}$ beträgt und unter der Voraussetzung, dass geeignete Sonnenschutzmaßnahmen umgesetzt werden, sollen beim Überschreiten einer Lufttemperatur im Raum von $+26^{\circ} \mathrm{C}$ zusätzliche Maßnahmen, wie unten aufgeführt, ergriffen werden. In seltenen Fällen kann es durch Arbeiten bei über $+26^{\circ} \mathrm{C} z \mathrm{u}$ einer Gefährdung der Gesundheit kommen. In diesen Fällen ist die Gefährdungsbeurteilung anzupassen und über weitere Maßnahmen zu entscheiden. Dies ist der Fall, wenn z.B.

- schwere körperliche Arbeit zu verrichten ist, - besondere Arbeits- oder Schutzbekleidung getragen werden muss, die die Wärmeabgabe stark behindert oder

- gesundheitlich Vorbelastete und besonders schutzbedürftige Beschäftigte (z.B. Jugendliche, Ältere, Schwangere, stillende Mütter) im Raum tätig sind.

Wird im Arbeitsraum die Lufttemperatur von $+30^{\circ} \mathrm{C}$ überschritten, sind nachfolgend aufgeführte wirksame Maßnahmen zu ergreifen, die die Beanspruchung der Beschäftigten verringern. Dabei gehen technische und organisatorische Maßnahmen den personenbezogenen Maßnahmen vor. 


\author{
Maßnahmen bei Überschreitung der Raum- \\ temperatur von $+26^{\circ} \mathrm{C}$ (und bei vorliegenden \\ besonderen Gegebenheiten) und von $+30^{\circ} \mathrm{C}$ \\ gemäß der Arbeitsstättenregel (ASR) 3.5
- effektive Steuerung des Sonnenschutzes (z.B. Jalou- sien auch nach der Arbeitszeit geschlossen halten)
v effektive Steuerung der Lüftungseinrichtungen (z.B. Nachtauskühlung)
- Reduzierung der inneren thermischen Lasten (z.B. elektrische Geräte nur bei Bedarf betreiben)
- Lüftung in den frühen Morgenstunden
- Nutzung von Gleitzeitregelungen zur Arbeitszeitver- lagerung
- Lockerung der Bekleidungsregelungen
- Bereitstellung geeigneter Getränke (z.B. Trinkwasser)

Wenn im Innenraum eine Lufttemperatur von $+35^{\circ} \mathrm{C}$ überschritten wird, so ist der betreffende Raum laut ASR 3.5 „Raumtemperatur“ in dieser Zeit ohne folgende Maßnahmen nicht als nicht als Arbeitsraum geeignet. Dies kann jedoch abgewendet werden, wenn folgende Maßnahmen ergriffen werden:

- technische Maßnahmen(z.B. Luftduschen, Wasserschleier),

- organisatorische Maßnahmen (z.B. Entwärmungsphasen) oder

- persönliche Schutzausrüstungen (z.B. Hitzeschutzkleidung)

Durch technische Maßnahmen, die die Lufttemperatur reduzieren, darf die absolute Luftfeuchte nicht erhöht werden. Für Baustellen gelten abweichende/ergänzende Anforderungen.

\subsubsection{Maßnahmen zum Schutz der Beschäftigten}

\section{Kühlung der Arbeitsumgebung}

Die Kühlung der Arbeitsumgebung ist der effektivste Weg, um Beschäftige vor Hitze zu schützen (Morris et al. 2020). Um negative Fol- gen für die Klimaerwärmung zu verhindern, sollte auf klimafreundliche Kühlanlagen gesetzt werden, wie beispielsweise Heiz- und Kühldeckensysteme, die im Sommer statt heißem kaltes Wasser transportieren. Von dieser Kühlung profitieren jedoch nur Beschäftige in Innenräumen und nicht diejenigen, die aufgrund ihrer Tätigkeit direkt der Hitze ausgesetzt sind, wie in der Landwirtschaft oder im Baugewerbe.

\section{Kleidung}

Eine angepasste Kleidung wird nach dem Kühlen der Arbeitsumgebung als wichtigster Schutz der Beschäftigten angesehen. Dabei ist das Tragen von Kühlwesten besonders effektiv. Aber auch die Nutzung von atmungsaktiver und luftiger Kleidung ermöglicht eine verbesserte Wärmeabfuhr über die Haut. Für Beschäftigte, die im Freien arbeiten und direkter Sonneneinstrahlung ausgesetzt sind, empfiehlt sich lichtreflektierende, helle Arbeitskleidung (Morris et al. 2020).

\section{Akklimatisation}

Mit gezielten Maßnahmen lässt sich die Toleranz gegenüber Hitze vor Beginn der Arbeit erhöhen (Aoyagi et al. 1997). Hauptsächlich durch eine Steigerung der körperlichen Fitness und eine größere Flüssigkeits- und Elektrolytaufnahme vor dem Arbeitseinsatz kann die Anpassungsfähigkeit der körpereigenen Thermoregulation verbessert werden. Dadurch ist es möglich, dass Hitzebelastungen deutlich länger ertragen werden können. Nach etwa zehn Tagen Akklimatisation wird prinzipiell mit einem ersten ausreichenden Schutz gerechnet und nach etwa vier Wochen ist der Körper in der Regel vollständig akklimatisiert (Piekarski u. Zerlett 1993). Jedoch sollte der Akklimatisationszustand nicht unterbrochen werden, denn laut DGAUM (2012) wird bei einer Unterbrechung 
II Gesundheitliche Auswirkungen des Klimawandels und Herausforderungen

für die medizinische Versorgung in Deutschland

von einer Woche der Akklimatisationsgrad um $50 \%$ verringert.

\section{Kühlen des Körpers}

Kaltes Wasser kann dem Körper eine angenehme Abkühlung ermöglichen und ist eine einfache und kostengünstige Möglichkeit des Hitzeschutzes. Dabei ist es für die Beschäftigten bereits entlastend, kühles Wasser über die Unterarme fließen zu lassen (Morris et al. 2020).

\section{Flüssigkeitshaushalt}

Um die eigene Gesundheit und Leistungsfähigkeit zu erhalten, sollte ein Arbeitnehmer an einem Hitzearbeitsplatz auf regelmäßige und ausreichend Flüssigkeitsaufnahme achten. Der durch Schwitzen entstandene Flüssigkeitsverlust sollte durch entsprechende Trinkmengen ausgeglichen werden, auch wenn der Betroffene kein Durstgefühl hat (sog. „preventive drinking“) (DGAUM 2012).

\section{Elektrolyte}

$\mathrm{Zu}$ Beginn der Arbeit an einem Hitzearbeitsplatz kann es aufgrund fehlender Anpassung des eigenen Körpers zu einem Elektrolytmangel kommen. Dieser reguliert sich jedoch mit zunehmender Anpassung, sodass bei ausreichender Zufuhr über die Nahrung auf eine ausgeprägte Supplementierung weitgehend verzichtet werden kann (DGAUM 2012). Ist der Akklimatisationsstatus noch unzureichend, kann mit einer Elektrolytlösung (zum Beispiel aus der Apotheke oder ein gestrichener TL Salz auf ein Liter Wasser) vor der Arbeitsschicht supplementiert werden.

\section{Energiebedarf/Ernährung}

Eine Erhöhung der Energiezufuhr durch eine Anpassung der Ernährung ist im Allgemeinen nicht notwendig, da der Energiebedarf hauptsächlich von der Arbeitsschwere abhängt (DGAUM 2012).

\section{Arbeitsorganisation}

Die Arbeitsschwere sollte an das Klima angepasst werden. Wie oben bereits beschrieben, sollte bei entsprechender Hitzebelastung am Arbeitsplatz versucht werden, die daraus entstehende Belastung für die Beschäftigten durch organisatorische Arbeitsschutzmaßnahmen zu verringern. Beispiele hierfür sind oben dargestellt. Leitlinienkonform kann im trockenen Klima ,bei einer Lufttemperatur von $35^{-}-45^{\circ} \mathrm{C}$ eine Unterbrechung von 15 oder 20 min pro Stunde notwendig sein. In den Arbeitspausen muss dem erwärmten Körper in geeigneter Umgebung (Schatten oder kühlere Bereiche) Gelegenheit zur Abkühlung gegeben werden“ (DGAUM 2012). Wenn (Berufs-)Kleidung getragen wird, die eine isolierende Wirkung hat, soll diese dann abgelegt, oder zumindest geöffnet werden.

\subsection{Zusammenfassung der Relevanz für Deutschland}

Die thermische Hitzebelastung am Arbeitsplatz wird sich vor dem Hintergrund der klimatischen Veränderung in den nächsten Jahren noch erhöhen. Dabei ist die Hitzebelastung abhängig von mehreren Faktoren wie Umgebungstemperatur, Art der Tätigkeit, individuelle Physiologie und Fitness sowie sozioökonomischer Situation. Studien zeigen neben den negativen Folgen für die Gesundheit der Beschäftigten auch negative Auswirkungen auf deren Leistungsfähigkeit und die Wirtschaftsleistung. 
Präventionsmaßnahmen sind für Deutschland im Rahmen der Leitlinien der wissenschaftlichen medizinischen Fachgesellschaften veröffentlicht und werden durch weitere praxisnahe Empfehlungen aus der Wissenschaft ergänzt. Dabei ist eine Kombination aus mehreren Maßnahmen sicherlich der effektivste Weg, um die Beschäftigten vor Hitze am Arbeitsplatz zu schützen.

Vor dem Hintergrund der Anzahl von Arbeitsunfähigkeitsmeldungen aufgrund von Hitzeerkrankungen ist zu diskutieren, ob bereits ausreichende Präventionsmaßnahmen am Arbeitsplatz umgesetzt werden.

Die zunehmende Betroffenheit Deutschlands in Bezug auf thermische Hitze am Arbeitsplatz sollte sich zum einen im „Vorbereitet sein" auf Hitzewellen, zum anderen aber auch in einer für die jeweilige Arbeitsstätte passgenauen Handlungsempfehlung im Falle einer Hitzewelle widerspiegeln.

Die Hitzebelastung am Arbeitsplatz hat Auswirkungen auf Gesundheit und Produktivität und sollte als Problem der öffentlichen Gesundheit anerkannt werden. Es sind (interdisziplinäre) Maßnahmen erforderlich, um die Auswirkungen angesichts des Klimawandels und des zu erwarteten Anstiegs des Hitzestresses abzuschwächen.

\section{Literatur}

an der Heiden M, Buchholz U, Uphoff H (2020) Schätzung der Zahl hitzebedingter Sterbefälle infolge der Hitzewelle 2018. UMID - UMWELT + MENSCH INFORMATIONSDIENST, 77-90

an der Heiden M, Muthers S, Niemann H, Buchholz U, Grabenhenrich L, Matzarakis A (2019) Schätzung hitzebedingter Todesfälle in Deutschland zwischen 2001 und 2015. Bundesgesundheitsblatt - Gesundheitsforschung - Gesundheitsschutz 62, 571-579

Aoyagi Y, McLellan TM, Shephard RJ (1997) Interactions of physical training and heat acclimation. The thermophysiology of exercising in a hot climate. Sports medicine (Auckland, N.Z.) 23, 173-210

Best S, Caillaud C, Thompson M (2012) The effect of ageing and fitness on thermoregulatory response to high-intensity exercise. Scand I Med Sci Sports 22, e29-37
Binazzi A, Levi M, Bonafede M, Bugani M, Messeri A, Morabito M, Marinaccio A, Baldasseroni A (2019) Evaluation of the impact of heat stress on the occurrence of occupational injuries: Meta-analysis of observational studies. Am | Ind Med 62, 233-243

Bissolli P, Deutschländer T, Imbery F, Lefebvre C, Blahak J, Fleckenstein R, Breyer |, Rocek M, Kreienkamp F, Rösner S, Schreiber K (2019) Hitzewelle Juli 2019 in Westeuropa - neuer nationaler Rekord in Deutschland. URL: www.dwd.de/DE/leistungen/besondereereignisse/temperatur/20190801_hitzerekord_juli2019.pdf?__blob=publicationFile\&v=2 (abgerufen am: 01.03.2021)

Brasseur GP, Jacob D, Schuck-Zöller S (2017) Klimawandel in Deutschland: Entwicklung, Folgen, Risiken und Perspektiven. Springer Spektrum Heidelberg

Bundesanstalt für Arbeitsschutz und Arbeitsmedizin (BAuA) (2018) Technische Regel für Arbeitsstätten ASR A3.5. URL: https:// www.baua.de/DE/Angebote/Rechtstexte-und-Technische-Regeln/Regelwerk/ASR/pdf/ASR-A3-5.pdf?_blob=publicationFile\&v=5 (abgerufen am 02.03.2021)

Bundesgesundheitsministerium (BMG) (2018a) Angaben zu den Geschäftsergebnissen der GKV bezüglich der Leistungsfälle und Tage. URL: https://www.bundesgesundheitsministerium. de/themen/krankenversicherung/zahlen-und-fakten-zurkrankenversicherung/geschaeftsergebnisse.html\#c10337 (abgerufen am: 01.03.2021)

Bundesgesundheitsministerium (BMG) (2018b) Arbeitsunfähigkeit: Fälle und Tage nach Diagnosen

Bundesministerium für Umwelt (BMU) (2013) Repräsentativumfrage zu Umweltbewusstsein und Umweltverhalten im Jahr 2012. Umweltforschungsplan des Bundesministeriums für Umwelt, Naturschutz und Reaktorsicherheit (BMU). Förderkennzeichen 37111711

Bundesministerium für Umwelt (BMU), Umweltbundesamt (UBA) (2017) Umweltbewusstsein in Deutschland 2016 - Ergebnisse einer repräsentativen Bevölkerungsumfrage

Casanueva A, Kotlarski S, Fischer AM, Flouris AD, Kjellstrom T, Lemke B, Nybo L, Schwierz C, Liniger MA (2020) Escalating environmental summer heat exposure-a future threat for the European workforce. Regional Environmental Change 20,40

Cuddy MLS (2004) The effects of drugs on thermoregulation. AACN Advanced Critical Care 15, 238-253

Deutsche Gesellschaft für Arbeitsmedizin und Umweltmedizin e.V. (DGAUM) (2012) Leitlinie Arbeit unter klimatischer Belastung: Hitze. URL: www.awmf.org/leitlinien/detail/ll/002-039.html (abgerufen am: 01.03.2021)

Deutsche Gesetzliche Unfallversicherung e.V. (DGUV) (2010) Beurteilung des Raumklimas. Gesund und fit im Kleinbetrieb

Deutsche Gesetzliche Unfallversicherung e.V. (DGUV) (2016) DGUV Information 215-510 Beurteilung des Raumklimas - Handlungshilfe für kleine und mittlere Unternehmen Berlin

Dorman LE, Havenith G (2009) The effects of protective clothing on energy consumption during different activities. European Journal of Applied Physiology 105, 463-470 
Flouris AD, Dinas PC, Ioannou LG, Nybo L, Havenith G, Kenny GP, Kjellstrom T (2018) Workers' health and productivity under occupational heat strain: a systematic review and meta-analysis. Lancet Planet Health 2, e521-e531

Gabriel KM, Endlicher WR (2011) Urban and rural mortality rates during heat waves in Berlin and Brandenburg, Germany. Environmental Pollution 159, 2044-2050

Gao C, Kuklane K, Östergren PO, Kjellstrom T (2018) Occupational heat stress assessment and protective strategies in the context of climate change. International Journal of Biometeorology $62,359-371$

Gubernot DM, Anderson GB, Hunting KL (2015) Characterizing occupational heat-related mortality in the United States, 20002010: an analysis using the Census of Fatal Occupational Injuries database. Am I Ind Med 58, 203-211

HARVARD Kennedy School (2018) When the heat is on, student learning suffers. URL: https://www.hks.harvard.edu/announcements/when-heat-student-learning-suffers (abgerufen am 01.03.2021)

Herrmann A, Haefeli WE, Lindemann U, Rapp K, Roigk P, Becker C (2019) Epidemiology and prevention of heat-related adverse health effects on elderly people. Zeitschrift fur Gerontologie und Geriatrie 52, 487-502

Jacklitsch B, Williams W, Musolin K, Coca A, Kim J-H, Turner N (2016) Occupational exposure to heat and hot environments: revised criteria 2016 106. URL: www.cdc.gov/niosh/docs/2016-106/ pdfs/2016-106.pdf?id=10.26616/NIOSHPUB2016106 (abgerufen am 01.03.2021)

Johnson RJ, Sánchez-Lozada LG, Newman LS, Lanaspa MA, Diaz HF, Lemery I, Rodriguez-Iturbe B, Tolan DR, Butler-Dawson I, Sato Y, Garcia G, Hernando AA, Roncal-Jimenez CA (2019) Climate Change and the Kidney. Ann Nutr Metab 74 Suppl 3, 38-44

Kalisch Ellett LM, Pratt NL, Le Blanc VT, Westaway K, Roughead EE (2016) Increased risk of hospital admission for dehydration or heat-related illness after initiation of medicines: a sequence symmetry analysis. Journal of Clinical Pharmacy and Therapeutics 41, 503-507

Kenny GP, Notley SR, Flouris AD, Grundstein A (2020) Climate Change and Heat Exposure: Impact on Health in Occupational and General Populations. In: Adams W, Jardine I (eds) Exertional Heat Illness. Springer, Cham

Kenny GP, Wilson TE, Flouris AD, Fujii N (2018) Heat exhaustion. Handbook of Clinical Neurology 157. Elsevier Amsterdam, 0xford, Cambridge

Kenny GP, Yardley I, Brown C, Sigal RJ, Jay 0 (2010) Heat stress in older individuals and patients with common chronic diseases. Cmaj 182, 1053-1060

Kiesel EK, Hopf YM, Drey M (2018) An anticholinergic burden score for German prescribers: score development. BMC geriatrics 18, 239

Kjellstrom T, Briggs D, Freyberg C, Lemke B, Otto M, Hyatt 0 (2016) Heat, Human Performance, and Occupational Health: A Key Issue for the Assessment of Global Climate Change Impacts. Annual Review of Public Health 37, 97-112

Kjellstrom T, Freyberg C, Lemke B, Otto M, Briggs D (2018) Estimating population heat exposure and impacts on working people in conjunction with climate change. International Journal of Biometeorology 62, 291-306

Kjellstrom T, Lemke B, Lee I (2019) Workplace Heat: An increasing threat to occupational health and productivity. Am I Ind Med 62, 1076-1078

Klenk ), Becker C, Rapp K (2010) Heat-related mortality in residents of nursing homes. Age and Ageing 39, 245-252

Knowlton K, Rotkin-Ellman M, King G, Margolis HG, Smith D, Solomon G, Trent R, English P (2009) The 2006 California heat wave: impacts on hospitalizations and emergency department visits. Environ Health Perspect 117, 61-67

Levi M, Kjellstrom T, Baldasseroni A (2018) Impact of climate change on occupational health and productivity: a systematic literature review focusing on workplace heat. Med Lav 109, 163-179

Lundgren K, Kuklane K, Gao C, Holmér I (2013) Effects of heat stress on working populations when facing climate change. Ind Health 51, 3-15

Mangoni AA, Kholmurodova F, Mayner L, Hakendorf P, Woodman RJ (2017) The Concomitant Use of Diuretics, Non-Steroidal Anti-Inflammatory Drugs, and Angiotensin-Converting Enzyme Inhibitors or Angiotensin Receptor Blockers (Triple Whammy), Extreme Heat, and In-Hospital Acute Kidney Injury in Older Medical Patients. Advances in Therapy 34, 2534-2541

Mangoni AA, Kim S, Hakendorf P, Mayner L, Woodman RJ (2016) Heat waves, drugs with Anticholinergic effects, and outcomes in older hospitalized adults. Journal of the American Geriatrics Society 64, 1091-1096

Masson-Delmotte V, Zhai P, Pörtner H-0, Roberts D, Skea I, Shukla PR, Pirani A, Moufouma-Okia W, Péan C, Pidcock R, Connors S, Matthews JBR, Chen Y, Zhou X, Gomis MI, Lonnoy E, Maycock T, Tignor M, Waterfield T (2019) IPCC 2018: Global warming of $1.5^{\circ} \mathrm{C}$ An IPCC Special Report on the impacts of global warming of $1.5^{\circ} \mathrm{C}$ above pre-industrial levels and related global greenhouse gas emission pathways, in the context of strengthening the global response to the threat of clima. URL: https://www.ipcc.ch/sr15/ (abgerufen am 09.03.2021)

Matthies F, Bickler G, Marin NC, Hales S (2008) Heat-health Action Plans. Guidance. World Health Organization Europe Copenhagen, Denmark. URL: https://www.euro.who.int/_data/assets/ pdf_file/0006/95919/E91347.pdf (abgerufen am 09.03.2021)

Matthies-Wiesler F (2019) Policy Brief für Deutschland. URL: www. bundesaerztekammer.de/fileadmin/user_upload/downloads/pdf-Ordner/Pressemitteilungen/20191114_Klimawandel/3_Lancet_Countdown_Policy_brief_for_Germany_German_v01b.pdf (abgerufen am 01.03.2021)

Meade RD, Akerman AP, Notley SR, McGinn R, Poirier P, Gosselin P, Kenny GP (2020) Physiological factors characterizing heatvulnerable older adults: A narrative review. Environ Int 144, 105909

Mora C, Counsell CW, Bielecki CR, Louis LV (2017) Twenty-seven ways a heat wave can kill you: Deadly heat in the era of climate change. Circulation: Cardiovascular Quality and Outcomes 10(11), e004233

Morabito M, Messeri A, Noti P, Casanueva A, Crisci A, Kotlarski S, Orlandini S, Schwierz C, Spirig C, Kingma BRM, Flouris AD, 
Nybo L (2019) An Occupational Heat-Health Warning System for Europe: The HEAT-SHIELD Platform. Int | Environ Res Public Health 16(16), 2890

Morris NB, Jay 0, Flouris AD, Casanueva A, Gao C, Foster J, Havenith G, Nybo L (2020) Sustainable solutions to mitigate occupational heat strain - An umbrella review of physiological effects and global health perspectives. Environmental Health: A Global Access Science Source 19, 1-24

Nawrot TS, Staessen JA, Fagard RH, van Bortel LM, Struijker-Boudier HA (2005) Endothelial function and outdoor temperature. Eur | Epidemiol 20, 407-410

Näyhä S (2005) Environmental temperature and mortality. International Journal of Circumpolar Health 64, 451-458

Nordon C, Martin-Latry K, Roquefeuil L de, Latry P, Bégaud B, Falissard B, Rouillon F, Verdoux H (2009) Risk of death related to psychotropic drug use in older people during the European 2003 heatwave: a population-based case-control study. The American Journal of Geriatric Psychiatry 17, 1059-1067

Piekarski C, Zerlett G (1993) Schädliche Einwirkungen und Noxen aus der Umwelt - Physikalische Einwirkungen. In: Classen M, Diehl V, Kochsiek K (Hrsg.) Innere Medizin. S. 865-876. Urban \& Schwarzenberg München, Wien, Baltimore

Rameezdeen R, Elmualim A (2017) The Impact of Heat Waves on Occurrence and Severity of Construction Accidents. International Journal of Environmental Research and Public Health 14, 70

Riley K, Wilhalme H, Delp L, Eisenman DP (2018) Mortality and Morbidity during Extreme Heat Events and Prevalence of Outdoor Work: An Analysis of Community-Level Data from Los Angeles County, California. Int I Environ Res Public Health 15, 580

Schoierer I, Mertes H, Wershofen B, Bose-0'Reilly S (2019) Trainingmodules on climate change, heat, and health for medical assistants and nurses in outpatient care. Bundesgesundheitsblatt - Gesundheitsforschung - Gesundheitsschutz 62, 620628

Senatsverwaltung für Stadtentwicklung und Wohnen (2015) Umweltatlas Berlin: 09.01 Umweltgerechtigkeit (Ausgabe 2015). URL: https://www.stadtentwicklung.berlin.de/umwelt/umweltatlas/d901 07.htm (abgerufen am 01.03.2021)

Spector JT, Masuda YJ, Wolff NH, Calkins M, Seixas N (2019) Heat Exposure and Occupational Injuries: Review of the Literature and Implications. Curr Environ Health Rep 6, 286-296

Statistisches Bundesamt (Destatis) (2020) Erwerbstätigkeit älterer Menschen. URL: https://www.destatis.de/DE/Themen/Querschnitt/Demografischer-Wandel/Aeltere-Menschen/erwerbstaetigkeit.html (abgerufen am 01.03.2021)

Steul K, Schade M, Heudorf U (2018) Mortality during heatwaves 2003-2015 in Frankfurt-Main - the 2003 heatwave and its implications. International Journal of Hygiene and Environmental Health 221, 81-86

Takaro TK, Knowlton K, Balmes JR (2013) Climate change and respiratory health: Current evidence and knowledge gaps. Expert Review of Respiratory Medicine 7, 349-361

Umweltbundesamt (UBA) (2019a) Klimawandel und Gesundheit Tipps für sommerliche Hitze und Hitzewellen. URL: https:// www.umweltbundesamt.de/publikationen/klimawandel-gesundheit-tipps-fuer-sommerliche-hitze (abgerufen am 01.03.2021)

Umweltbundesamt (UBA) (2019b) Monitoringbericht 2019 zur Deutschen Anpassungsstrategie an den Klimawandel. URL: https://www.umweltbundesamt.de/publikationen/umweltbundesamt-2019-monitoringbericht-2019-zur (abgerufen am 01.03.2021)

Universitätsklinikum Heidelberg (2020) DOSING: Informationen zur Arzneimittel-Anwendung \& -Sicherheit. URL: www.dosing. de (abgerufen am 01.03.2021)

Vanos |, Vecellio D|, Kjellstrom T (2019) Workplace heat exposure, health protection, and economic impacts: A case study in Canada. Am I Ind Med 62, 1024-1037

Vassallo SU, Delaney KA (1989) Pharmacologic effects on thermoregulation: mechanisms of drug-related heatstroke. Journal of Toxicology: Clinical Toxicology 27, 199-224

Watts N, Amann M, Ayeb-Karlsson S, Belesova K, Bouley T, Boykoff M, Byass P, Cai W, Campbell-Lendrum D, Chambers I, Cox PM, Daly M, Dasandi N, Davies M, Depledge M, Depoux A, Dominguez-Salas $P$, Drummond $P$, Ekins $P$, Flahault A, Frumkin $H$, Georgeson L, Ghanei M, Grace D, Graham H, Grojsman R, Haines A, Hamilton I, Hartinger S, Johnson A, Kelman I, Kiesewetter G, Kniveton D, Liang L, Lott M, Lowe R, Mace G, Odhiambo Sewe M, Maslin M, Mikhaylov S, Milner I, Latifi AM, Moradi-Lakeh M, Morrissey K, Murray K, Neville T, Nilsson M, Oreszczyn T, Owfi F, Pencheon D, Pye S, Rabbaniha M, Robinson E, Rocklöv |, Schütte S, Shumake-Guillemot J, Steinbach $\mathrm{R}$, Tabatabaei M, Wheeler N, Wilkinson P, Gong P, Montgomery H, Costello A (2018) The Lancet Countdown on health and climate change: from 25 years of inaction to a global transformation for public health. The Lancet 391, 581-630

Westaway K, Frank 0, Husband A, McClure A, Shute R, Edwards S, Curtis I, Rowett D (2015) Medicines can affect thermoregulation and accentuate the risk of dehydration and heat-related illness during hot weather. Journal of Clinical Pharmacy and Therapeutics 40, 363-367

Zheng G, Li K, Wang Y (2019) The Effects of High-Temperature Weather on Human Sleep Quality and Appetite. International Journal of Environmental Research and Public Health 16, 270 

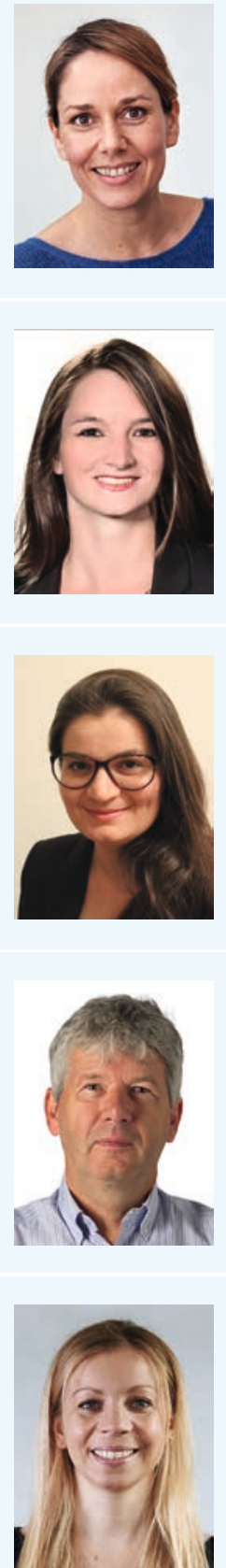

\section{Dr. phil. Julia Schoierer}

Julia Schoierer ist Medizinpädagogin und entwickelt am Institut für Arbeits-, Sozial- und Umweltmedizin des LMU-Klinikums Anpassungsmaßnahmen an den Klimawandel. Dabei ist die Hitze, als eine Auswirkung der klimatischen Veränderungen, in den Fokus ihrer Arbeit gerückt und damit der Schutz von Beschäftigen im Gesundheitswesen sowie von Risikogruppen.

\section{Hanna Mertes}

Master of Science in Public Health, beschäftigt sich bereits seit einigen Jahren mit den gesundheitlichen Auswirkungen von Umwelteinflüssen. Seit 2016 liegt ihr Fokus am Institut und Poliklinik für Arbeits-, Sozial- und Umweltmedizin (AG Globale Umwelt-Gesundheit) auf den gesundheitlichen Folgen des Klimawandels und der dahingehenden Schulung von Gesundheitsberufen.

\section{Katharina Deering, M.A.}

Seit Dezember 2016 am Institut und Poliklinik für Arbeits-, Sozial- und Umweltmedizin in der AG Globale Umwelt-Gesundheit tätig. Seitdem unterstützt sie Projekte zu den Auswirkungen des Klimawandels auf die menschliche Gesundheit.

\section{PD Dr. med. Stephan Böse-0'Reilly}

Habilitiert für den Bereich Umweltmedizin und Public Health. Als Kinder- und Jugendarzt interessiert er sich vor allem für die Zusammenhänge von Kind, Umwelt und Gesundheit. Er leitet die Arbeitsgruppe Globale Umwelt-Gesundheit am Institut und Poliklinik für Arbeits-, Sozial- und Umweltmedizin des LMU-Klinikums in München. Forschungsschwerpunkte sind gesundheitliche Folgen des Klimawandels, Quecksilberbelastungen im Goldbergbau, Bleibelastung von Kindern, globale Gesundheit und Umweltverschmutzung.

\section{Dr. med. Caroline Quartucci}

Fachärztin für Arbeitsmedizin am Institut für Arbeits-, Sozial- und Umweltmedizin des LMU-Klinikums. Im Rahmen ihrer Tätigkeit ist sie im Betriebsärztlichen Dienst des LMU-Klinikums für die arbeitsmedizinischen Vorsorgen und die Betreuung von über 10.000 Beschäftigten des Klinikums zuständig. Ihre Aufgabe ist es, den Arbeitgeber beim Arbeitsschutz und bei der Unfallverhütung in allen Fragen des Gesundheitsschutzes zu unterstützen, wozu auch die Einführung von Arbeitsverfahren und die Auswahl und Erprobung von Körperschutzmitteln gehören. 